\title{
EFFECTS OF ACOUSTIC WAVES ON SPECTRAL LINE PROFILES
}

Lawrence E. Cram

Sacramento Peak Observatory*

Sunspot, NM 88349, U.S.A.

\section{Abstract}

Almost all studies of spectral line formation in the presence of non-thermal velocity fields have been made assuming that the only effect of the velocity field is to produce a Doppler shift of the absorption and emission coefficients. However, a non-thermal velocity field will entail velocity-correlated fluctuations in temperature, pressure, level populations, and other parameters of the line formation problem. Using a time-dependent dynamical calculation describing the propagation of non-linear, radiatively-damped short period $(\mathrm{P}=30 \mathrm{~s})$ acoustic waves in the solar photosphere, Cram, Keil and Ulmschneider (1980) have shown that velocity-correlated fluctuations in state variables (particularly the temperature) may lead to important effects in line broadening, line shifts and asymmetries, and in line-shift oscillations. Upwardly propagating waves generally produce significant redshifts in the cores of mediumstrong Fe I lines, and the increased ratio of observed line shift to wave velocity amplitude would significantly modify the results of kinematic studies of high frequency line shifts such as those of Deubner (1976) and Keil (1980).

Cram (1980) has further explored dynamical effects in the formation of Fe $I$ and Fe II lines by using the "microturbulence" limit, wherein an average is made over the phase of the wave before the transfer equation is solved. Except for weak, high EP Fe II lines, the predicted solar lines are redshifted and show a "red" asymmetry. For a model of Arcturus the lines are often shifted to the blue, but it does not appear that this model can account for the observed differences between solar and Arcturan line asymmetries (Gray 1980).

References

Cram, L.E., 1980 (in preparation).

Cram, L.E., Keil, S.L., Ulmschneider, P., 1980, Ap.J. (in press). Deubner, F.-L., 1976, Astr. Ap. 51, 189.

Gray, D., 1980, Ap.J. (in press).

Keil, S.L., 1980, Ap.J. (submitted).

* Operated by the Association of Universities for Research in Astronomy, Inc. under contract AST-78-17292 with the National Science Foundation. 